\title{
A Holistic Ecosystem for Rural mHealth Applications and Lesson Learnt
}

\author{
Jacey-Lynn Minoi, IEEE Member, Mohd Raili Suhaili, and Alvin W Yeo, IEEE Member
}

\begin{abstract}
Delivering mHealth applications requires a mHealth ecosystem and this paper will present the development of an ecosystem of mHealth applications to be deployed at remote and rural villages that do not enjoy the same health benefits as in urban areas. Some evidence was found to indicate advantages of $\mathbf{m H e a l t h}$ applications in supporting healthy living and wellness behavioural change among the people. The fundamental principle of an ecosystem is to enhance the participation of all the stakeholders and to understand the big picture of the work process holistically. The stakeholders include developers, telecommunication providers, patients, caregivers, healthcare providers, the Ministry of Health and other government sectors. It is important to balance the commercial interests and the peoples' needs. For the last decade, commercialised mHealth applications are increasingly available to target heterogeneous audiences and address specific health needs. With that, research interest in mHealth is also increasing together with the complexity of the ecosystems that impact all the stakeholders. We will present the development of protocols and privacy from lessons learnt to increase the effectiveness of mHealth in practice and to allow better understanding for future development and employment. We have also conducted a pilot participatory study at four telecentres at rural areas in Malaysia using commercialised mHealth systems.
\end{abstract}

\section{INTRODUCTION}

According to a recent report [1], mHealth application is increasing in an exponential growth offering mobile healthcare solutions. mHealth is "the delivery of health care services via mobile communication devices." [2]. Its market is currently in the commercialisation stage whereby the revenue is generated by mHealth services and hardware sales. Despite the huge increase of sales, mHealth is still lacking of guidelines and regulations. These issues are significant barriers to creating an integrated mHealth solution application.

At present, many mHealth applications target only medical stakeholders, such doctors, nurses/caregivers and patients. Multiple health care providers (HCPs) could serve multiple patients every day or during hospitalisation. Small but growing evidence have shown that mHealth applications

Research supported by ISITI and MAXIS fund under grant no. UHSB/BAM2013/01

J. -L. Minoi is with the Faculty of Computer Science and Information Technology, Universiti Malaysia Sarawak, Malaysia (e-mail: jacey@fit.unimas.my).

M. R. Suhaili (M.D.) was with the Faculty of Medical and Health Sciences, Universiti Malaysia Sarawak. He is now with the Faculty of Medicine, SEGi University, Malaysia (e-mail: mrsuhaili@gmail.com).

A. W. Yeo is with the Institute of Social Informatics and Technological Innovation - Rural Informatics, Malaysia (e-mail: alvin@isiti.unimas.my). can improve diagnosis, patient information and hence could increase administrative efficiency [3].

We have conducted several community-based pilot participatory studies to different rural communities using two commercialised mHealth applications and an in-house mHealth system based on a proposed mHealth ecosystem. We employed these mHealth applications with emphasis on patients with hypertension and obesity. Questionnaire-based needs analysis and interviews were conducted. The patients were recruited amongst those who went for community health screening at the telecentres and clinics. With the support of the State Director of Health Department, participants were recruited also at the local clinics. All participants in this study were briefed and requested to sign an informed consent.

The concept of mHealth application is to store and send health reading data wirelessly to a cloud-based remote server. Doctors can view the data at regular basis remotely from a website. Throughout the process of the participatory study, we gave some thoughts on the future designs and interfaces of the mHealth applications with the current shortcomings with the stakeholders. We jointly work together with developers and telecommunication industries to answer the shortcomings of the designs, which later should elucidate which of the mHealth applications are beneficial for appropriate situations in terms of cost and speed for health care decision making while still engaging the rural communities.

The Malaysian Communications and Multimedia Commission (MCMC) is a regulatory body that aims to promote and regulate Internet access to applications and services environment to end-users. To date, there is 2,175 telecentres in Malaysia built for rural communities with access to computers and the Internet to gather information and communicate with others. We selected four telecentres and each at Kampung Serasot, Lubok Antu and Meradong in Sarawak Borneo and Yan Kechil, Kedah for this study.

The aims of our work are to determine the important issues facing the implementation of mHealth from the perspective of those working in health care, patients or healthy people, private mHealth developers, telecommunication industries; and to develop an ecosystem for mHealth applications for all stakeholders. The involvement of mHealth developers is necessary to diverse the design of the application with a clear focus on the technology and practice; telecommunication industries is to ensure persistent connectivity of the health services; health care sectors including health care practitioners (e.g. doctors, nurses) and policy makers (people at the federal health 